\title{
Moving Target Search Algorithm with Informational Distance Measures
}

\author{
Eugene Kagan and Irad Ben-Gal ${ }^{*}$
}

Department of Industrial Engineering, Tel Aviv University, Ramat Aviv, 69978, Israel

\begin{abstract}
We consider an Ishida and Korf Moving Target Search (MTS) algorithm with informational distance measures. Similarly to the previously defined Informational Learning Real-Time A* algorithm, the suggested algorithm acts on the set of partitions of the sample space, on which the probability mass function is defined. The information-based Rokhlin metric and its lower bound - Ornstein metric, give the necessary distance measures. We prove that similarly to the Ishida and Korf MTS algorithm, the proposed Informational MTS (IMTS) algorithm always terminates and finds the target. The comparison of the IMTS algorithm with known models shows that it outperforms known Markov decision process model of search with probabilistic and informational decision criteria. These findings help to construct a unified framework of search after both static and moving targets, and to bridge the gap between different search procedures that are related to both artificial intelligence and information theory.
\end{abstract}

Keywords: Informational planning and learning, real-time heuristic search, Rokhlin distance, entropy.

\section{INTRODUCTION}

The problem of search after a moving target is one of the oldest problems that appear in different tasks and applications. In general, this problem is formulated as follows. Consider a target, which is moving within a discrete domain, and a searcher looking for that target. At each step, the action available to the searcher is to check a sub-domain in order to determine whether the target is somewhere within this sub-domain or not. If the searcher finds the target in a sub-domain that contains only one point, then the procedure terminates. Otherwise, the target moves to another point of domain, and the search continues. It is assumed that the target is not informed about the searcher's behavior and, in contrast to game theoretic formulation, and is not aimed to reach any goal. The searcher's goal, in opposite, is to choose a sequence of sub-domains such that the search procedure terminates in a minimal expected number of steps. Such formulation of the search procedure is motivated by a grouptesting procedure formulated by Dorfman [1]. Although, in contrast to the original Dorfman group testing, which implies a search after a static target, the formulated problem considers a search after a moving target.

The problem of search after a moving target was considered by the use of different approaches and methods, especially by the use of optimization techniques $[2,3]$. In particular, under additional restrictions on the abilities of the searcher and the target, the problem was intensively studied in the framework of search and screening theory $[4,5]$. For the search in discrete space, this direction was continued by Eagle [6] and later by Singh and Krishnamurthy [7].

The other approach to the search problem was suggested by Ishida and Korf [8, 9]. Based on Korf's Learning

*Address correspondence to this author at the Department of Industrial Engineering, Tel Aviv University, Ramat Aviv, 69978, Israel; Tel: +972640-69-10; Fax: +972-640-69-10; E-mail: bengal@eng.tau.ac.il
Real-Time A* (LRTA*) algorithm of search after a static target [10], Ishida and Korf built the Moving Target Search (MTS) algorithm that implements the same principles as LRTA* algorithm and acts on a connected graph. Recent results on these algorithms were reviewed by Shimbo and Ishida [11]. The probabilistic methods into the framework of LRTA* was introduced by Koenig et al. [12, 13] who considered an implementation of the LRTA* algorithm over non-deterministic domains and suggested suitable adaptive algorithms that can be applied for the search for both static and moving targets. Further studies of the algorithms of such type resulted in a unified framework for general search procedures that was suggested by Bulitko et al. $[14,15]$ and by the IRCL Research group [16].

The informational version of the LRTA* algorithm that implements a search after a static target in probabilistic space was suggested by Kagan and Ben-Gal [17]. This Informational LRTA* (ILRTA*) algorithm follows the Dorfman group-testing procedure [1] although implements a representation of search process in the form of search over partitions of the domain. Such a representation is motivated by a Generalized Optimal Testing Algorithm (GOTA) [18], which creates suitable partitions of sample space according to the set of available tests, and by the parallelized Huffman searching procedure [19] that implements Huffman search [20] over a partition of samples space.

In the paper, we follow the line of the ILRTA* algorithm [18] and extend it to the search for a moving target.

The formulated algorithm applies a group-testing approach to the MTS algorithm and is based on informationtheoretic measures. It acts on the set of partitions of the sample space similarly to other group-testing approaches and, in contrast to the existing algorithms of search, uses the information-based Rokhlin distance [21] to measure the distances between the partitions. Since the used distance measure meets the metric requirements, unlike other informational measures such as the Kulback-Leibler (KL) 
distance [22], it can be applied to the MTS algorithm. Accordingly, we call the suggested algorithm as the Informational MTS (IMTS) algorithm. Unlike other search procedures with moving target, the IMTS algorithm can utilize side information regarding the distance estimation from the searcher to the target, e.g., by implementation of Ornstein distance measure [23].

A main goal of the paper is to finalize a development of the unified informational search framework, which was started by the ILRTA* algorithm [17]. In this paper, we formulate the IMTS algorithm and study its main properties. The work is organized as follows. In Section 2, we formulate a general group-testing model of the search process after a moving target and describe the Rokhlin and Ornstein metrics. Section 4 includes numerical studies of IMTS algorithm and simulated statistical comparisons between the IMTS algorithm, the ILRTA* algorithm (in the case of static target) [17] and the model of search with informational criterions based on Markov decision processes (MDP) [24]. In the Conclusion, we summarize the work and discuss its future directions. APPENDIX contains the proofs of the statements formulated in the main text.

\section{A GROUP-TESTING SCHEME OF SEARCH AND REQUIRED DISTANCE MEASURES}

\subsection{Dorfman Scheme of Search Over Partitions Space}

Let $X=\left\{x_{1}, x_{2}, \ldots, x_{n}\right\}$ be a finite sample space of $n$ points where the target can be located. According to the Dorfman scheme of group-testing [1], at each time moment $t=0,1,2, \ldots$, the searcher chooses a subset $A^{t} \subset X$ and observes it. It is assumed that the observation is perfect, hence, the obtained observation result $z^{t}$ is such that $z^{t}=1$ if the target is located in one of the points of $A^{t}$, and $z^{t}=0$ otherwise. All subsets $A \subset X$ that are available to the searcher's choice are included into a search space $\mathbf{X}$. If the searcher's choice of subsets $A$ is not restricted, then the search space can be considered as a power set $\mathbf{X}=2^{X}$ of a sample space $X$ that consists of all possible subsets of $X$ including the empty set $\varnothing$ and the set $X$ itself.

The formulated Dorfman search scheme represents a general search procedure that, under suitable restrictions, covers most cases of search after a static target. In addition, such a scheme can be directly applied to the search after a moving target that acts on a sample space. In such a case, location of the target at time $t$ is denoted by $x^{t} \in X$. We implement the Dorfman search scheme in the equivalent form of search over partitions set $\chi$ as it was previously implemented by the Informational Learning Real-Time A* (ILRTA*) algorithm of search for a static target [17] that generalizes GOTA [18] and Huffman search [20].

The general procedure of search after a moving target on the partitions set $\chi$ is formulated as follows [24, 25]. Assume that at each time moment $t$ the target selects a location $x^{t} \in X$, and the searcher obtains a perfect observation result $z^{t} \in\{0,1\}$. Selection of the set $A^{t} \in \mathbf{X}$ and its observation implies that if $z^{t}=\mathbf{1}\left(x^{t}, A^{t}\right)=1$, then $z^{t}=\mathbf{1}\left(x^{t}, X \backslash A^{t}\right)=0$ and vice versa. Hence, such a selection can be represented by the searcher's partition $\alpha^{t}=\left\{A^{t}, X \backslash A^{t}\right\}$, the target location at time moment $t$ can be represented by the target's partition $\tau^{t}=\left\{\left\{x^{t}\right\}, X \backslash\left\{x^{t}\right\}\right\}$. Accordingly, the Dorfman search procedure on the set $\chi$ of partitions of the sample space $X$ in the case of moving target can be formulated as follows [25]:

1. The target starts for the point $x^{0} \in X$ that corresponds to the partition $\tau^{0}=\left\{\left\{x^{0}\right\}, X \backslash\left\{x^{0}\right\}\right\} \in \chi$. At each time moment $t$, the target chooses a point $x^{t} \in X$ that corresponds to the partition $\tau^{t} \in \chi$.

2. At time moment $t$, the searcher chooses a partition $\alpha^{t} \in \chi$.

3. If $\alpha^{t}=\tau^{t}$, then the search terminates. Otherwise time increases to $t=t+1$, the target chooses the next point $x^{t+1} \in X$ and corresponding partition $\tau^{t+1} \in \chi$ and the search continues.

It is assumed that the searcher is not informed about the target's location, while the probability of the target's location in the points of sample space $X$ can be available. The goal of the searcher is to find a policy for choosing the partitions $\alpha^{t} \in \chi$ that guarantees termination of the search within a minimal expected number of steps.

In the next sections, we formulate an Informational Moving Target Search (IMTS) algorithm that implements the above presented search procedure. This algorithm uses distances between partitions that are described below.

\subsection{Rokhlin and Ornstein Distance Measures}

We start with the definitions of the Rokhlin [21] and Ornstein [23] distances between partitions. These distances were previously used in the implementation of the ILRTA* algorithm of search after a static target [17], and in the models of search after moving target [24, 25].

Let $X=\left\{x_{1}, x_{2}, \ldots, x_{n}\right\}$ be a finite sample space. Assume that for every time moment $t$ there is defined a probability mass function $p^{t}: X \rightarrow[0,1]$. Functions $p^{t}$ determine the probabilities $p^{t}\left(x_{i}\right)=\operatorname{Pr}\left\{x^{t}=x_{i}\right\}$ of the target being located in points $x_{i} \in X, i=1,2, \ldots, n$, at time moment $t$, where $\sum_{i=1}^{n} p^{t}\left(x_{i}\right)=1$. Probabilities $p^{t}\left(x_{i}\right)$ are called the location probabilities. If there is no available information on the target location, then, basing on the principle of the maximum entropy, it is assumed that the probabilities $p^{t}\left(x_{i}\right)$ are equal. Below, for an arbitrary fixed time moment, we omit index $t$ in the notation of function $p^{t}$. 
Let $\chi$ be a set of partitions of the sample space $X$ and let $\alpha=\left\{A_{1}, A_{2}, \ldots\right\} \in \chi, A_{i} \cap A_{j}=\varnothing, i \neq j, \cup_{A \in \alpha}=X$, be a partition of $X$. For a subset $A \in \alpha$, denote $p(A)=\sum_{x \in A} p(x)$.

The entropy of the partition $\alpha$ is defined as follows [26]:

$H(\alpha)=-\sum_{A \in \alpha} p(A) \log p(A)$,

where, following the information theory conventions [22], the logarithm is taken base 2 and it is assumed that $0 \log 0=0$.

Let $\quad \beta=\left\{B_{1}, B_{2}, \ldots\right\} \in \chi, \quad B_{i} \cap B_{j}=\varnothing, \quad i \neq j$, conditional entropy of partition $\alpha$ given partition $\beta$ is [21, 26]:

$H(\alpha \mid \beta)=-\sum_{B \in \beta} \sum_{A \in \alpha} p(A, B) \log p(A \mid B)$,

where $p(A, B)=p(A \cap B)$ and $p(A \mid B)=p(A \cap B) / p(B)$.

Finally, the Rokhlin distance between the partitions $\alpha$ and $\beta$ of the sample space is defined as follows [21]:

$d(\alpha, \beta)=H(\alpha \mid \beta)+H(\beta \mid \alpha)$

where conditional entropy is defined by eq. (2).

Since the Rokhlin metric (3) is closely related with joint entropy and mutual information [17, 25], we also refer to such metric as to informational distance measure. In the next considerations, the following metric properties of the Rokhlin distance [26] will be used:

1. $d(\alpha, \beta) \geq 0$;

2. $\quad d(\alpha, \alpha)=0$

3. $d(\alpha, \beta) \leq d(\alpha, \xi)+d(\xi, \beta)$ for any partition $\xi$ of $X$.

The Ornstein metric gives another distance measure between partitions of the sample space $X$ with a probability mass function $p$. This metric, which is also called the Ornstein distance, was originally suggested as a measure of difference between stochastic processes and it implies that the partitions are ordered. The metric is defined as follows [23]:

$d_{\text {Orn }}(\alpha, \beta)=p\left(X \backslash \cup_{i=1}^{k}\left(A_{i} \cap B_{i}\right)\right), \alpha, \beta \in \chi$,

where $A_{i} \in \alpha, B_{i} \in \beta$, and $k=\max (|\alpha|,|\beta|)$. Here $|\cdot|$ is the cardinality of the partition. If $|\alpha|>|\beta|$, then $\beta$ is completed by empty sets, and if $|\beta|>|\alpha|$, then empty sets are added to $\alpha$. In other words, if $|\alpha|>|\beta|$, then from both partitions the first $|\beta|$ elements are taken, and if $|\beta|>|\alpha|$, then there are taken the first $|\alpha|$ elements.

Similar to the Rokhlin distance, the Ornstein distance meets the metric requirements [23]. For the Rokhlin and Ornstein distances the admissibility property holds.

Lemma 1 [17]. If $\alpha$ and $\beta$ are partitions of the same space $X$ with probability function $p$, then $d_{\text {Orn }}(\alpha, \beta) \leq d(\alpha, \beta)$.

In the next section we apply the above-presented metrics to the formulated search problem, and implement them in the Informational Moving Target Search (IMTS) algorithm.

\section{THE INFORMATIONAL MTS ALGORITHM AND ITS MAIN PROPERTIES}

The suggested Informational Moving Target Search algorithm (IMTS algorithm) implements above presented general Dorfman search procedure on the set $\chi$ of partitions of the sample space $X$. This algorithm is a special case of the Ishida and Korf Moving Target Search (MTS) algorithm [8, 9]. Similar to the ILRTA* algorithm of search after a static target [17] both the required distances and the neighborhood of the states in the IMTS algorithm are defined using informational Rokhlin and Ornstein metrics.

Let, as above, $X=\left\{x_{1}, x_{2}, \ldots, x_{n}\right\}$ be a sample space with corresponding location probabilities $p\left(x_{i}\right)$, $i=1,2, \ldots, n, \quad \sum_{i=1}^{n} p\left(x_{i}\right)=1$, and let $\chi$ be the set of all possible partitions of $X$ that includes both the trivial partition $\theta=\{X, \varnothing\}$ and the discrete partition $\gamma=\left\{\left\{x_{1}\right\},\left\{x_{2}\right\}, \ldots,\left\{x_{n}\right\}\right\}$.

Assume that for every pair of partitions $\alpha, \beta \in \chi$ one can define the Rokhlin distance $d(\alpha, \beta)$ and a distance estimation $\tilde{d}(\alpha, \beta)$, such that they satisfy

$\tilde{d}(\alpha, \beta) \leq d(\alpha, \beta), \alpha, \beta \in \chi$.

Property (5) is called the admissibility property. In the light of Lemma 1, it can be assumed that $\tilde{d}$ is the Ornstein metric, thus $\tilde{d}(\alpha, \beta)=d_{\text {Orn }}(\alpha, \beta)$. Note that, without loss of generality, it is assumed that for the discrete partition $\gamma$ and every $\alpha \in \chi$ estimation, $\tilde{d}(\alpha, \gamma)=0$ and the algorithm can still be applied.

The neighborhood of the states is a key concept in the MTS algorithm since the distance estimation between states is accurate only between the neighbors. For the MTS algorithm $[8,9]$ the neighborhood of the state is defined as a set of its successors in the graph over which the search is conducted. In the suggested IMTS algorithm, in contrast, the neighbors are defined by the use of the proposed informational distances between partitions from $\chi$ as follows. Let $r$ be a positive constant, $0 \leq r \leq H(X)$, where $H(X)$ is the entropy of sample space $X$. For any partition $\alpha \in \chi$, we say that $N(\alpha, r) \subset \chi$ is the set of neighbors of $\alpha$ if $N(\alpha, r)$ meets the following requirements: 
1. $\alpha \notin N(\alpha, r)$;

2. for every partition $\xi \in N(\alpha, r)$ it holds true that $\tilde{d}(\xi, \alpha) \leq r \leq d(\xi, \alpha)$.

Notice that in contrast to the neighborhood definition that appears in Ishida and Korf's MTS algorithm [8, 9], this one essentially uses the distances and distance estimations between the partitions - a situation that is often justified in practical applications.

Denote by $\tau$ (with suitable indices) the partitions chosen from $\chi$ by the target (which is often defined by a single-point subset and its complementary subset) and denote by $\alpha$ (with suitable indices) the partitions chosen from $\chi$ by the searcher.

In the proposed algorithm, the target's movements are restricted so that for every movement from $\tau$ to $\tau_{\text {cur }}$ it holds true that $d\left(\tau_{\text {cur }}, \tau\right)<r$.

Let $\theta$ and $\vartheta$ be arbitrary initial searcher's and target's partitions, respectively, and let $\tilde{d}_{0}(\alpha, \tau), \alpha, \tau \in \chi$, be the initial distance estimations. In the worst case, from the searcher's viewpoint it may be assumed that $\tilde{d}_{0}(\alpha, \tau)=0$ for all $\alpha, \tau \in \chi$. The IMTS algorithm is formulated as follows.

Algorithm IMTS. Given $\mathrm{r}, \theta, \vartheta, \tilde{d}_{0}(\alpha, \tau), \alpha, \tau \in \chi$,

1. Init distance estimations by $\tilde{d}_{0}(\alpha, \tau), \alpha, \tau \in \chi$.

2. Init searcher's current partition $\alpha_{c u r}$ by the initial partition $\theta$.

3. Init target's current partition $\tau_{\text {cur }}$ by the initial partition $\vartheta$.

4. While $d\left(\alpha_{\text {cur }}, \tau_{\text {cur }}\right) \neq 0$ do

\section{Searcher's Turn}

a. Choose next partition $\alpha_{\text {next }}$ (ties are resolved arbitrarily): $\alpha_{\text {next }} \leftarrow \arg \min _{\alpha \in N\left(\alpha_{\text {cur }}, r\right)}\left\{\tilde{d}\left(\alpha, \tau_{\text {cur }}\right)\right\}$.

b. Set distance estimation for current partition $\alpha_{\text {cur }}$ :

$\tilde{d}\left(\alpha_{c u r}, \tau_{c u r}\right) \leftarrow \max \left\{\tilde{d}\left(\alpha_{c u r}, \tau_{c u r}\right), \quad r+\min _{\alpha \in N\left(\alpha_{c u r}, r\right)}\left[\tilde{d}\left(\alpha, \tau_{c u r}\right)\right]\right\}$.

c. Move to next partition:

$\alpha_{\text {cur }} \leftarrow \alpha_{\text {next }}$.

\section{Target's Turn:}

\section{d. Target moves from partition $\tau$ to partition $\tau_{\text {cur }}$ :}

$\tau_{\text {cur }} \leftarrow \tau$.

e. $\quad$ Searcher updates estimation for partition $\tau$ :

$\tilde{d}\left(\alpha_{c u r}, \tau\right) \leftarrow \max \left\{\tilde{d}\left(\alpha_{\text {cur }}, \tau\right), \quad \tilde{d}\left(\alpha_{\text {cur }}, \tau_{\text {cur }}\right)-r\right\}$.

End while.

The actions of the IMTS algorithm are illustrated by Fig. (1). In the figure, triangles denote the searcher's partitions and the star denotes the target's partition.

In the figure we consider the case where the searcher location is represented by the partition $\alpha_{i}$ and the target location is represented by the partition $\tau_{i}$.

The suggested IMTS algorithm follows the principles of the ILRTA* algorithm of search after a static target [17]. In addition, as mentioned above, the IMTS algorithm can be viewed as an informational version of the MTS algorithm [8, 9], in which distances and distance estimations are strictly defined using the information-theoretic metrics. On the other side, the original MTS algorithm can be viewed as a particular case of the suggested IMTS algorithm applied to an equiprobable sample space.

Before the next consideration, let us formulate the following standard lemma of correctness. This lemma guarantees that the introduced requirements and restrictions do not contradict.

Lemma 2 (of correctness). The requirements for the neighborhood and the target's movement are correct in the terms of distances and distance estimations.

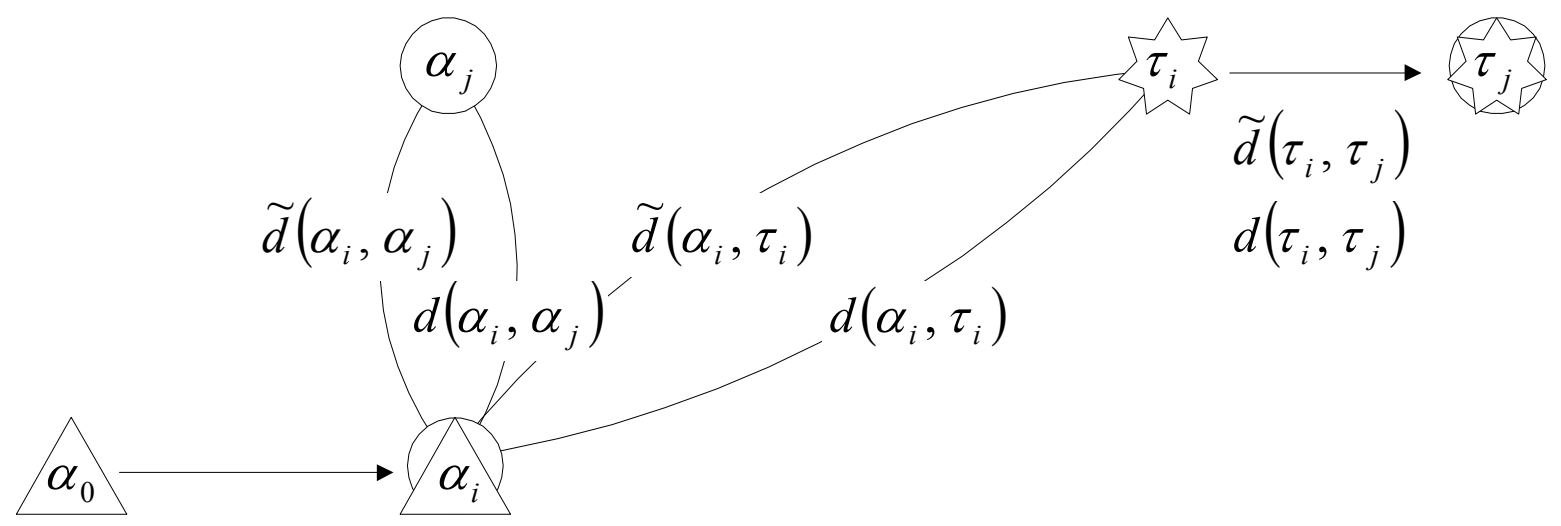

Fig. (1). Actions of the Informational Moving Target Search (IMTS) algorithm. 
The proof is given in APPENDIX.

Now let us show that for the IMTS algorithm the basic properties of the MTS algorithm [8, 9] still hold. The provided below new proofs of these properties are based on the proposed informational metrics and do not depend on the proofs regarding MTS algorithm.

The next lemma guarantees that the IMTS algorithm preserves the admissibility property (5) of distances $d$ and distance estimations $\tilde{d}$.

Lemma 3. The IMTS algorithm preserves the admissibility of estimation values $\tilde{d}(\alpha, \tau),(\alpha, \tau) \in \chi \times \chi$, i.e., throughout the IMTS algorithm's trial it holds true that $\tilde{d}(\alpha, \tau) \leq d(\alpha, \tau)$.

The main result regarding IMTS algorithm is the following.

Theorem 1. If for every pair $(\alpha, \tau) \in \chi \times \chi$ it the admissibility property $\tilde{d}_{0}(\alpha, \tau) \leq d(\alpha, \tau)$ holds and that it holds true that $d\left(\tau^{\prime}, \tau\right)-\tilde{d}\left(\tau^{\prime}, \tau\right)<d\left(\alpha^{\prime}, \alpha\right)-\tilde{d}\left(\alpha^{\prime}, \alpha\right)$, $\alpha, \alpha^{\prime}, \tau, \tau^{\prime} \in \chi$, then the IMTS algorithm always terminates.

The proofs of Lemma 3 and Theorem 1 are given in APPENDIX.

The above formulated statements show that suggested IMTS algorithm has the similar properties as the conventional MTS algorithm [8,9]. Although, notice that the requirements of Theorem 1, which guarantee the termination of the suggested IMTS algorithm, are weaker than the requirements for the MTS algorithm. Also, similarly to the ILRTA* algorithm [17] of search for a static target, the suggested IMTS algorithm allows an application of different probability measures on the sample space $X$, that makes it rather useful for different tasks.

\section{NUMERICAL STUDY AND SIMULATION RESULTS}

In this section we present the results of some numerical simulations of search with the IMTS algorithm. The simulations address the search after a target governed by different moving rules. In all simulations, we use the Rokhlin metric as a measure of a distance between partitions and as its estimates. Each simulation contained 1000 replications of the search algorithm. These replications were executed by a suitable $\mathrm{C}++$ program.

In the simulations, we considered three types of target's movements:

1. Static target;

2. Markovian target with uniformly distributed transition probabilities,

3. Brownian target with uniformly distributed transition probabilities.

When considering the target's movement we followed the conventional approach that implements Markovian or Brownian movements of the target. These models correspond to real-life constrains and assumptions of moving targets, e.g., movement continuity and a limit memory of real targets on a terrain $[27,28]$.

We analyzed the search procedures with different values of the radius $r$ that determines the neighborhoods of the searcher's partitions and the searcher's expectations regarding a "length" of the target's steps. Various implementations of the proposed algorithm were tested under different search spaces, including for a search over large databases [29, 30], as well as for a search by autonomous mobile agents [27, 28, 31]. Let us note that for large domains, a group-testing procedure after static targets, such as the ILRTA* algorithm [17], can be applied at the beginning of search; such offline procedures provide an initial partitioning of the sample space with initial location probabilities of the target. At later stages of the search, the propose IMTS procedure can be used over a smaller domain, while updating the partitions and their probabilities.

Using an offline search procedure at the beginning of the search is very appealing, since most of the offline grouptesting procedures (e.g., the ILRTA* or Huffamn) reach the entropy lower-bound for the expected number of tests. This bound, in the worst case, is close to the entropy of the location probability plus one. This fact implies that even if the search space contains for example 10,000 points, it will require only $\left[\log _{2} 10,000\right]=14$ binary group-tests to reach the vicinity of the target. Taking these observations into account, in the simulated study we focus on the last stage of the search near the target, while ignoring the relatively fixed (and optimal) number of test rounds till this area is reached. Below, we present the results of straightforward application of the algorithm for a search in the last stages of the search over a sample space $X$ of the size $|X|=9$ points.

The first simulation trials were conducted for the search after a static target. The results of the simulations of search for a static target with different values of the radius $r$ are presented in Table $\mathbf{1}$.

Table 1. Search for a Static Target

\begin{tabular}{|c|c|c|c|c|}
\hline \multirow{2}{*}{ Radius $\boldsymbol{r}$} & \multicolumn{4}{|c|}{ Number of Steps } \\
\cline { 2 - 5 } & Min & Max & Mean & Std. Dev. \\
\hline \hline$r=0.1$ & 1 & 15 & 8.255 & 4.584 \\
\hline$r=0.5$ & 1 & 15 & 8.235 & 4.592 \\
\hline$r=1$ & 1 & 15 & 8.284 & 4.575 \\
\hline$r=2$ & 1 & 15 & 8.262 & 4.576 \\
\hline$r=3$ & 1 & 15 & 8.239 & 4.576 \\
\hline
\end{tabular}

The results presented in Table $\mathbf{1}$ show that the average number of search steps slightly depends on the radius $r$ but it quite robust with respect to this parameter. The Welch's $t$ test $(\alpha=0.05)$ shows that the differences between the maximum mean value obtained for $r=1$ and minimal mean value obtained for $r=0.5$ is not significant ( $t_{\text {observed }}=0.239$ and $t_{\text {stat }}=1.960$ ). 
The next simulation trials were conducted for the search after a Markovian target. The results of the simulations for different values of the radius $r$ are presented Table 2 .

Table 2. Search for a Markovian Target

\begin{tabular}{|c|c|c|c|c|}
\hline \multirow{2}{*}{ Radius $\boldsymbol{r}$} & \multicolumn{4}{|c|}{ Number of Steps } \\
\cline { 2 - 5 } & Min & Max & Mean & Std. Dev. \\
\hline \hline$r=0.1$ & 1 & 76 & 9.264 & 11.021 \\
\hline$r=0.5$ & 1 & 75 & 9.220 & 10.916 \\
\hline$r=1$ & 1 & 76 & 8.737 & 10.267 \\
\hline$r=2$ & 1 & 79 & 8.767 & 10.392 \\
\hline$r=3$ & 1 & 78 & 8.957 & 10.502 \\
\hline
\end{tabular}

For the search after Markovian target, the average number of search steps reaches its maximum for $r=0.1$ and minimum for $r=1$, however, according to the Welch's $t$-test ( $\alpha=0.05$ ), this difference is not significant $\left(t_{\text {observed }}=1.106\right.$ and $t_{\text {stat }}=1.960$ ).

The final simulation trials were conducted for the search after a Brownian target. The results of the simulations for different values of the radius $r$ are presented in Table 3 .

Table 3. Search for a Brownian Target

\begin{tabular}{|c|c|c|c|c|}
\hline \multirow{2}{*}{ Radius $\boldsymbol{r}$} & \multicolumn{4}{|c|}{ Number of Steps } \\
\cline { 2 - 5 } & Min & Max & Mean & Std. Dev. \\
\hline \hline$r=0.1$ & 1 & 72 & 10.766 & 11.503 \\
\hline$r=0.5$ & 1 & 78 & 10.887 & 11.786 \\
\hline$r=1$ & 1 & 80 & 10.615 & 11.221 \\
\hline$r=2$ & 1 & 72 & 10.759 & 11.244 \\
\hline$r=3$ & 1 & 78 & 10.558 & 11.269 \\
\hline
\end{tabular}

For a search after a Brownian target, the average number of search steps reaches its maximum for $r=0.5$ and minimum for $r=3$. As for the previous cases, according to the Welch's $t$-test ( $\alpha=0.05)$, here again the difference is not significant $\left(t_{\text {observed }}=0.639\right.$ and $\left.t_{\text {stat }}=1.960\right)$ and the procedure is found to be robust.

The last set of simulations compares the suggested IMTS algorithm with popular Markov Decision Processes (MDP) models of search [32] with both probabilistic and informational criterions. The MDP model and its variants rely on an expectation regarding the target's locations at the next time steps, which determined the searcher's selections [24].

We conducted such simulations of the IMTS algorithm that uses the same partitions as MPD models. In particular, we used partitions space $\chi$ that includes only such partitions that consists of two subsets and one of the subsets includes only one point. The results of simulation trials of the IMTS algorithm with restricted partitions are presented in Table 4.
Table 4. Search for with Restricted Partitions

\begin{tabular}{|c|c|c|c|c|c|}
\hline \multirow{2}{*}{$\begin{array}{c}\text { Parameter } \boldsymbol{r} \text { and Type } \\
\text { of Target Movement }\end{array}$} & \multicolumn{4}{|c|}{ Number of Steps } \\
\cline { 3 - 6 } & Min & Max & Mean & Std. Dev. \\
\hline \hline \multirow{3}{*}{$r=0.1$} & Static target & 1 & 8 & 4.486 & 2.253 \\
\cline { 2 - 6 } & Markovian target & 1 & 26 & 4.875 & 4.089 \\
\cline { 2 - 6 } & Brownian target & 1 & 33 & 4.987 & 4.607 \\
\hline \multirow{3}{*}{$r=1.0$} & Static target & 1 & 8 & 4.521 & 2.252 \\
\cline { 2 - 6 } & Markovian target & 1 & 26 & 4.627 & 3.849 \\
\cline { 2 - 6 } & Brownian target & 1 & 33 & 5.005 & 4.675 \\
\hline
\end{tabular}

The results presented in Table 4 show that in spite of a large difference between the maximal number of the steps required for finding the static and the moving targets (for Markovian target the number of step is more than three times greater than for the static target and for Brownian target it is more than four times greater than for the static target), the average number of search steps are rather close. This fact demonstrates that the suggested algorithm provides a successful search both for static and for moving targets, and, consequently, can be considered as an effective solution of the old problem [33], which requires extending classical two-cell Pollock model of search [34] to the search over arbitrary number of cells.

The results presented in Table 4 were compared with those obtained by the MDP models of search [24]. Note that both the search by IMTS algorithm and the search using MDP models include an expectation about the next target's location. The MDP model is based on the known target's transition probabilities, while the IMTS algorithm uses constant expectation and assumes that the target applies the step, which is the worst for the searcher.

Using the Welch's $t$-tests $\left(\alpha=0.05, t_{\text {stat }}=1.960\right)$, we compared the results obtained by the IMTS algorithm with $r=1.0$ with the results obtained by using the MDP model with maximum expected information criterion. The results of the comparison are the following:

1. Static target: $t_{\text {observed }}=3.385$; the expected number of search steps 4.521 obtained by the IMTS algorithm is significantly less than the expected number of search steps 4.928 obtained by using the MDP model.

2. Markovian target: $t_{\text {observed }}=4.303$; the expected number of search steps 4.627 obtained by the IMTS algorithm is significantly less than the expected number of search steps 5.535 obtained by using the MDP model.

3. Brownian target: $t_{\text {observed }}=1.333$; the expected number of search steps 5.005 obtained by the IMTS algorithm is statistically equivalent to the expected number of search steps 4.747 obtained by using the MDP model.

As it is expected, the results of the comparison show that in the case of the static target and in the case of Markovian 
target, the IMTS algorithm, which applies the worst-case expectation, demonstrates better results than the MDP model of search, which expects with certainty the target movement at the next step. In the case of Brownian target search, both algorithms demonstrate statistically equivalent results, since such a target cannot stay in its current location and the worst-case expectation is close to the expectation based on the transition probabilities matrix.

Finally, we compared the results of the application of the IMTS algorithm to the search after a static target with the best results of such a search obtained by the ILRTA* algorithm [17]. The Welch's $t$-tests ( $\left.\alpha=0.05, t_{\text {stat }}=1.960\right)$ give the following results:

1. ILRTA* algorithm with Huffman neighborhood, the Rokhlin distance and zero estimations (average number of steps is 5.575) and IMTS algorithm with $r=1.0$ and general neighborhood (average number of steps is 8.284) were found to be significantly different, $t_{\text {observed }}=17.339$.

2. ILRTA* algorithm with Huffman neighborhood, the Rokhlin distance and zero estimations (average number of steps is 5.575) and IMTS algorithm with $r=1.0$ and restricted neighborhood (average number of steps is 4.521) were found to be significantly different, $t_{\text {observed }}=11.396$.

3. ILRTA* algorithm with unrestricted neighborhood, the Rokhlin distance and the Ornstein estimations (average number of steps is 2.790) and IMTS algorithm with $r=1.0$ and restricted neighborhood (average number of steps is 4.521) were found to be

$$
\text { significantly different, } t_{\text {observed }}=18.723 \text {. }
$$

The results of the above comparisons show that the effectiveness of the search after a static target depends on the type of neighborhood and on the estimation scheme. A search based on the ILRTA* algorithm without estimation and with an Huffman neighborhood gives significantly better results than the search by using the IMTS algorithm with an unrestricted neighborhood and the worst estimation. Nevertheless, the outcome of the same ILRTA* algorithm is significantly worse than the outcome of the IMTS algorithm with the same estimation and a restricted neighborhood.

\section{CONCLUSIONS}

In this paper, we considered the problem of search after a moving target. The target moves over a discrete sample space, and the action, which is available to the searcher, is to check a subset of locations and to determine whether the target is located somewhere in this subset or not. The procedure terminates if the searcher finds the target in a subset, which contains only one point. The goal is to find a sequence of subsets such that the search procedure terminates in a minimal average number of steps.

The proposed Informational Moving Target Search (IMTS) algorithm follows a group testing theory approach. This algorithm is based on the MTS algorithm and, in contrast to known search methods, allows taking into account available side information about possible target's location, if such information exists.

The suggested IMTS algorithm acts over the set of partitions of the sample space. Thus, the searcher can take into account both the chosen subset of the sample space and the set of remaining subsets. We use the Rokhlin metric and,

Table 5. Comparative Summary of the IMTS Algorithm and Other Known Algorithm of Search

\begin{tabular}{|c|c|c|c|c|}
\hline & & Static Target & Markovian Target & Brownian Target \\
\hline \multirow{2}{*}{ 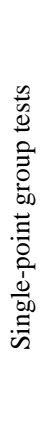 } & 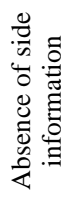 & $\begin{array}{l}\text { ILRTA* algorithm } \\
\text { IMTS algorithm }\end{array}$ & $\begin{array}{c}\text { Probabilistic MDP } \\
\text { Informational MDP } \\
\text { IMTS algorithm }\end{array}$ & $\begin{array}{c}\text { Probabilistic MDP } \\
\text { Informational MDP } \\
\text { IMTS algorithm }\end{array}$ \\
\hline & 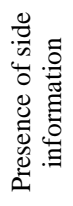 & $\begin{array}{l}\text { ILRTA* algorithm } \\
\text { IMTS algorithm }\end{array}$ & $\begin{array}{c}\text { Probabilistic MDP } \\
\text { Informational MDP } \\
\text { IMTS algorithm }\end{array}$ & $\begin{array}{c}\text { Probabilistic MDP } \\
\text { Informational MDP } \\
\text { IMTS algorithm }\end{array}$ \\
\hline \multirow{2}{*}{ 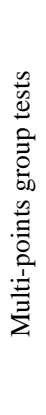 } & 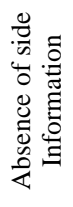 & $\begin{array}{l}\text { ILRTA* algorithm } \\
\text { IMTS algorithm }\end{array}$ & $\begin{array}{l}\text { Informational MDP } \\
\text { IMTS algorithm }\end{array}$ & $\begin{array}{l}\text { Informational MDP } \\
\text { IMTS algorithm }\end{array}$ \\
\hline & 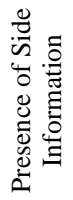 & $\begin{array}{l}\text { ILRTA* algorithm } \\
\text { IMTS algorithm }\end{array}$ & $\begin{array}{l}\text { Informational MDP } \\
\text { IMTS algorithm }\end{array}$ & $\begin{array}{l}\text { Informational MDP } \\
\text { IMTS algorithm }\end{array}$ \\
\hline
\end{tabular}


if need, the Ornstein metric as distance measures between the partitions and as admissible distance estimations.

The features of the suggested IMTS algorithm are analyzed theoretically and it is proven that it always terminates with finding the target. Numerical simulations show that the suggested IMTS algorithm outperforms the known MDP model of search with probabilistic and informational decision criteria. The results of the comprehensive study of the suggested IMTS and other known procedures of search are summarized in the Table $\mathbf{5}$. Bold letters mark those algorithms that provide the best results in terms of minimal average number of search steps.

The suggested algorithm can be applied to a number of practical problems. In addition to straightforward search, its key ideas were rather fruitful for path-planning and navigation of mobile robots [27, 28, 35] and for consideration of data-mining problems [36]. The algorithm can be also be applied for a paging process in cellular networks and for other applications that allow a formulation in the form of stochastic local search.

Finally, the IMTS algorithm can be generalized for a search after a number of targets and to a search by multiple searchers. Following preliminary results regarding such a search after a static target by multiple searchers [37], these studies are remained for future research.

\section{CONFLICT OF INTEREST}

The authors confirm that this article content has no conflict of interest.

\section{ACKNOWLEDGEMENTS}

Declared none.

\section{APPENDIX}

Proof of Lemma 2. Let searcher's partition be $\alpha_{i-1}$ and target's partition be $\tau_{i-1}$. Assume that the searcher moves to partition $\alpha_{i} \in N\left(\alpha_{i-1}, r\right)$ and that the target moves to the partition $\tau_{i}$. Since $\alpha_{i} \in N\left(\alpha_{i-1}, r\right)$, it holds true that $\tilde{d}\left(\alpha_{i-1}, \alpha_{i}\right) \leq r \leq d\left(\alpha_{i-1}, \alpha_{i}\right)$, and according to the assumed restriction for target's movement it holds true that $\tilde{d}\left(\tau_{i-1}, \tau_{i}\right) \leq d\left(\tau_{i-1}, \tau_{i}\right)<r$.

We need to show that the inequality $d\left(\alpha_{i}, \tau_{i}\right)-\tilde{d}\left(\alpha_{i}, \tau_{i}\right) \leq d\left(\alpha_{i-1}, \tau_{i}\right)-\tilde{d}\left(\alpha_{i-1}, \tau_{i}\right)$ is consistent.

If $\tau_{i} \in N\left(\alpha_{i-1}, r\right)$, then $\tilde{d}\left(\alpha_{i}, \tau_{i}\right)=0$, and since estimation $\tilde{d}$ is a metric, it follows that $\alpha_{i}=\tau_{i}$. Thus $d\left(\alpha_{i}, \tau_{i}\right)=0$, as well. Hence, the required inequality becomes $0 \leq d\left(\alpha_{i-1}, \tau_{i}\right)-\tilde{d}\left(\alpha_{i-1}, \tau_{i}\right)$, that follows from the definition of distance estimation.

Let $\tau_{i} \notin N\left(\alpha_{i-1}, r\right)$. Then $\quad \tilde{d}\left(\alpha_{i-1}, \tau_{i}\right)>r \quad$ and $d\left(\alpha_{i-1}, \tau_{i}\right)>r$. Therefore, we have: $d\left(\alpha_{i}, \tau_{i}\right) \leq d\left(\alpha_{i-1}, \alpha_{i}\right)+d\left(\alpha_{i-1}, \tau_{i}\right), r \leq d\left(\alpha_{i-1}, \alpha_{i}\right)$,

$r<d\left(\alpha_{i-1}, \tau_{i}\right)$

$\tilde{d}\left(\alpha_{i}, \tau_{i}\right) \leq \tilde{d}\left(\alpha_{i-1}, \alpha_{i}\right)+\tilde{d}\left(\alpha_{i-1}, \tau_{i}\right), r \geq d\left(\alpha_{i-1}, \alpha_{i}\right)$,

$r<\tilde{d}\left(\alpha_{i-1}, \tau_{i}\right)$.

Subtracting the second inequality from the first, we obtain:

$d\left(\alpha_{i}, \tau_{i}\right)-\tilde{d}\left(\alpha_{i}, \tau_{i}\right) \leq d\left(\alpha_{i-1}, \alpha_{i}\right)+$

$d\left(\alpha_{i-1}, \tau_{i}\right)-\tilde{d}\left(\alpha_{i-1}, \alpha_{i}\right)-\tilde{d}\left(\alpha_{i-1}, \tau_{i}\right)$

that is correct since $d\left(\alpha_{i-1}, \alpha_{i}\right) \geq r, \quad d\left(\alpha_{i-1}, \tau_{i}\right)>r$, $\tilde{d}\left(\alpha_{i-1}, \alpha_{i}\right) \leq r$ and $\tilde{d}\left(\alpha_{i-1}, \tau_{i}\right)>r$. Q.E.D.

Proof of lemma 3. Let $\tilde{d}_{0}(\alpha, \tau) \leq d(\alpha, \tau)$ for every available pair $(\alpha, \tau) \in \chi \times \chi$. We need to show that following steps of the IMTS algorithm it is guaranteed that $\tilde{d}\left(\alpha_{\text {cur }}, \tau\right) \leq d\left(\alpha_{\text {cur }}, \tau\right)$.

Let us consider the actions.

Searcher's turn. If it holds true that $\tilde{d}\left(\alpha_{\text {cur }}, \tau_{\text {cur }}\right) \geq \min _{\xi \in N\left(\alpha_{\text {cur }}, r\right)}\left\{\tilde{d}\left(\xi, \tau_{\text {cur }}\right)+r\right\}$, then by step 4.b of the algorithm the estimation updating is $\tilde{d}\left(\alpha_{\text {cur }}, \tau_{\text {cur }}\right) \leftarrow \tilde{d}\left(\alpha_{\text {cur }}, \tau_{\text {cur }}\right)$, and the admissibility holds since $\tilde{d}\left(\alpha_{\text {cur }}, \tau_{\text {cur }}\right) \leq d\left(\alpha_{\text {cur }}, \tau_{\text {cur }}\right)$.

Let $\tilde{d}\left(\alpha_{\text {cur }}, \tau_{\text {cur }}\right)<\min _{\xi \in N\left(\alpha_{c u r}, r\right)}\left\{\tilde{d}\left(\xi, \tau_{c u r}\right)+r\right\}$, and define $\alpha_{\min }=\arg \min _{\xi \in N\left(\alpha_{c u r}, r\right)}\left\{\tilde{d}\left(\xi, \tau_{\text {cur }}\right)\right\}$. Then, by step 4.b we have $\tilde{d}\left(\alpha_{\text {cur }}, \tau_{\text {cur }}\right)<\tilde{d}\left(\alpha_{\text {min }}, \tau_{\text {cur }}\right)+r$ and we need to show that $r+\tilde{d}\left(\alpha_{\min }, \tau_{c u r}\right) \leq d\left(\alpha_{c u r}, \tau_{c u r}\right)$. If $\tau_{c u r} \in N\left(\alpha_{c u r}, r\right)$ then $\tilde{d}\left(\alpha_{\min }, \tau_{\text {cur }}\right)=0, \quad d\left(\alpha_{\text {cur }}, \tau_{\text {cur }}\right)=d\left(\alpha_{\text {cur }}, \alpha_{\text {min }}\right) \quad$ and the required inequality becomes $r \leq d\left(\alpha_{c u r}, \alpha_{\min }\right)$, that holds according to definition of neighborhood. Let $\tau_{\text {cur }} \notin N\left(\alpha_{\text {cur }}, r\right)$. So $r<\tilde{d}\left(\alpha_{\text {cur }}, \tau_{\text {cur }}\right)$. Using the triangle inequality, we obtain:

$r+\tilde{d}\left(\alpha_{\min }, \tau_{\text {cur }}\right) \leq d\left(\alpha_{\text {cur }}, \alpha_{\text {min }}\right)+d\left(\alpha_{\text {min }}, \tau_{c u r}\right)$. Recalling that $r \leq d\left(\alpha_{\text {cur }}, \alpha_{\min }\right)$ and $\tilde{d}\left(\alpha_{\min }, \tau_{\text {cur }}\right) \leq d\left(\alpha_{\text {min }}, \tau_{\text {cur }}\right)$ we obtain the required inequality.

Target's turn. If it holds true that $\tilde{d}\left(\alpha_{\text {cur }}, \tau\right) \geq \tilde{d}\left(\alpha_{\text {cur }}, \tau_{\text {cur }}\right)-r$; then the estimation updating is $\tilde{d}\left(\alpha_{\text {cur }}, \tau\right) \leftarrow \tilde{d}\left(\alpha_{\text {cur }}, \tau\right)$, and since $\tilde{d}\left(\alpha_{\text {cur }}, \tau\right) \leq d\left(\alpha_{\text {cur }}, \tau\right)$ the admissibility holds. Let $\tilde{d}\left(\alpha_{\text {cur }}, \tau\right)<\tilde{d}\left(\alpha_{\text {cur }}, \tau_{\text {cur }}\right)-r$. Then we need to show that $\tilde{d}\left(\alpha_{\text {cur }}, \tau_{\text {cur }}\right)-r \leq d\left(\alpha_{\text {cur }}, \tau\right)$. According to the triangle inequality, we have $d\left(\alpha_{\text {cur }}, \tau\right)+d\left(\tau, \tau_{\text {cur }}\right) \geq d\left(\alpha_{\text {cur }}, \tau_{\text {cur }}\right), \quad$ and $\quad$ since 
$d\left(\tau, \tau_{\text {cur }}\right)<r$ and $d\left(\alpha_{\text {cur }}, \tau_{\text {cur }}\right) \geq \tilde{d}\left(\alpha_{\text {cur }}, \tau_{\text {cur }}\right)$, holds true that $d\left(\alpha_{\text {cur }}, \tau\right)+r \geq d\left(\alpha_{\text {cur }}, \tau_{\text {cur }}\right) \geq \tilde{d}\left(\alpha_{\text {cur }}, \tau_{\text {cur }}\right)$, that is the required inequality. Q.E.D.

Proof of Theorem 1. Let the searcher's partition be $\alpha_{i-1}$ and the target's partition be $\tau_{i-1}$, and suppose that the searcher moves to partition $\alpha_{i} \in N\left(\alpha_{i-1}, r\right)$ and the target moves to the partition $\tau_{i}$. We need to show that after updating it is guaranteed that

$d\left(\alpha_{i}, \tau_{i}\right)-\tilde{d}\left(\alpha_{i}, \tau_{i}\right)<d\left(\alpha_{i-1}, \tau_{i-1}\right)-\tilde{d}\left(\alpha_{i-1}, \tau_{i-1}\right)$.

Since the target was not found on the previous step, it follows that $\tau_{i-1} \notin N\left(\alpha_{i-1}, r\right), \quad \tilde{d}\left(\alpha_{i-1}, \tau_{i-1}\right)>r \quad$ and $d\left(\alpha_{i-1}, \tau_{i-1}\right)>r$. If $\tau_{i} \in N\left(\alpha_{i-1}, r\right)$, then $\tilde{d}\left(\alpha_{i}, \tau_{i}\right)=0$, and since estimation $\tilde{d}$ is a metric it leads to $\alpha_{i}=\tau_{i}$ and the algorithm terminates.

Let $\tau_{i} \notin N\left(\alpha_{i-1}, r\right)$. Then $\quad \tilde{d}\left(\alpha_{i-1}, \tau_{i}\right)>r \quad$ and $d\left(\alpha_{i-1}, \tau_{i}\right)>r$. Suppose that the searcher moved from $\alpha_{i-2}$ to $\alpha_{i-1}$ and the target move from $\tau_{i-1}$ to $\tau_{i}$. Denote by $\tilde{d}^{\prime}(\cdot, \cdot)$ the estimation values obtained after updating.

Let the searcher's next partition be $\alpha_{i}=\arg \min _{\xi \in N\left(\alpha_{i-1}, r\right)}\left\{\tilde{d}\left(\xi, \tau_{i-1}\right)+r\right\}$. The estimation updating at the searcher's move is $\tilde{d}^{\prime}\left(\alpha_{i}, \tau_{i-1}\right)=\max \left\{\tilde{d}\left(\alpha_{i-1}, \tau_{i-1}\right), \quad \tilde{d}\left(\alpha_{i}, \tau_{i-1}\right)+r\right\}$, and the estimation updating at the target's move is $\tilde{d}^{\prime}\left(\alpha_{i}, \tau_{i}\right)=\max \left\{\tilde{d}^{\prime}\left(\alpha_{i}, \tau_{i-1}\right), \tilde{d}\left(\alpha_{i}, \tau_{i}\right)-r\right\}$. Then we have the following cases for updating.

(a) $\tilde{d}^{\prime}\left(\alpha_{i}, \tau_{i-1}\right)=\tilde{d}\left(\alpha_{i-1}, \tau_{i-1}\right), \tilde{d}^{\prime}\left(\alpha_{i}, \tau_{i}\right)=\tilde{d}^{\prime}\left(\alpha_{i}, \tau_{i-1}\right)$.

According to the triangle inequality for distance and distance estimation, we obtain:

$$
\begin{aligned}
& \tilde{d}^{\prime}\left(\alpha_{i}, \tau_{i-1}\right) \leq \tilde{d}\left(\alpha_{i-1}, \alpha_{i}\right)+\tilde{d}\left(\alpha_{i}, \tau_{i-1}\right), \\
& \tilde{d}^{\prime}\left(\alpha_{i}, \tau_{i}\right) \leq \tilde{d}\left(\tau_{i-1}, \tau_{i}\right)+\tilde{d}\left(\alpha_{i}, \tau_{i-1}\right) ; \\
& d\left(\alpha_{i-1}, \tau_{i-1}\right) \leq d\left(\alpha_{i-1}, \alpha_{i}\right)+d\left(\alpha_{i}, \tau_{i-1}\right), \\
& d\left(\alpha_{i}, \tau_{i}\right) \leq d\left(\tau_{i-1}, \tau_{i}\right)+d\left(\alpha_{i}, \tau_{i-1}\right) .
\end{aligned}
$$

Subtraction of the first inequalities from the second ones gives:

$$
\begin{aligned}
& d\left(\alpha_{i-1}, \tau_{i-1}\right)-\tilde{d}^{\prime}\left(\alpha_{i}, \tau_{i-1}\right) \leq d\left(\alpha_{i 1}, \alpha_{i}\right)- \\
& \tilde{d}\left(\alpha_{i-1}, \alpha_{i}\right)+d\left(\alpha_{i}, \tau_{i-1}\right)-\tilde{d}\left(\alpha_{i}, \tau_{i-1}\right) \\
& d\left(\alpha_{i}, \tau_{i}\right)-\tilde{d}^{\prime}\left(\alpha_{i}, \tau_{i}\right) \leq d\left(\tau_{i-1}, \tau_{i}\right)- \\
& \tilde{d}\left(\tau_{i-1}, \tau_{i}\right)+d\left(\alpha_{i}, \tau_{i-1}\right)-\tilde{d}\left(\alpha_{i}, \tau_{i-1}\right)
\end{aligned}
$$

So, $\quad d\left(\alpha_{i}, \tau_{i}\right)-\tilde{d}\left(\alpha_{i}, \tau_{i}\right)<d\left(\alpha_{i-1}, \tau_{i-1}\right)-\tilde{d}\left(\alpha_{i-1}, \tau_{i-1}\right)$ is guaranteed, if it holds true that $d\left(\tau_{i-1}, \tau_{i}\right)-\tilde{d}\left(\tau_{i-1}, \tau_{i}\right)<d\left(\alpha_{i-1}, \alpha_{i}\right)-\tilde{d}\left(\alpha_{i-1}, \alpha_{i}\right)$, that is the assumption of the theorem.

(b) $\tilde{d}^{\prime}\left(\alpha_{i}, \tau_{i-1}\right)=\tilde{d}\left(\alpha_{i-1}, \tau_{i-1}\right), \tilde{d}^{\prime}\left(\alpha_{i}, \tau_{i}\right)=\tilde{d}\left(\alpha_{i}, \tau_{i}\right)-r$.

Since $\tilde{d}\left(\alpha_{i}, \tau_{i}\right) \leq \tilde{d}\left(\tau_{i-1}, \tau_{i}\right)+\tilde{d}\left(\alpha_{i}, \tau_{i-1}\right)$, for

$\tilde{d}^{\prime}\left(\alpha_{i}, \tau_{i}\right)=\tilde{d}\left(\alpha_{i}, \tau_{i}\right)-r$ it also holds true that $\tilde{d}^{\prime}\left(\alpha_{i}, \tau_{i}\right) \leq \tilde{d}\left(\tau_{i-1}, \tau_{i}\right)+\tilde{d}\left(\alpha_{i}, \tau_{i-1}\right)$. Thus, this case is the same as the previous one.

(c) $\tilde{d}^{\prime}\left(\alpha_{i}, \tau_{i-1}\right)=\tilde{d}\left(\alpha_{i}, \tau_{i-1}\right)+r, \tilde{d}^{\prime}\left(\alpha_{i}, \tau_{i}\right)=\tilde{d}^{\prime}\left(\alpha_{i}, \tau_{i-1}\right)$.

According to Lemma 3, the IMTS algorithm preserves admissibility of estimations. Hence

$\tilde{d}^{\prime}\left(\alpha_{i}, \tau_{i-1}\right)=\tilde{d}\left(\alpha_{i}, \tau_{i-1}\right)+r \leq d\left(\alpha_{i}, \tau_{i-1}\right)$.

Since $\tilde{d}\left(\alpha_{i-1}, \alpha_{i}\right) \leq r$ and

$\tilde{d}^{\prime}\left(\alpha_{i}, \tau_{i-1}\right)=\tilde{d}\left(\alpha_{i}, \tau_{i-1}\right)+r \leq \tilde{d}\left(\alpha_{i-1}, \alpha_{i}\right)+\tilde{d}\left(\alpha_{i}, \tau_{i-1}\right), \quad$ we have $\tilde{d}\left(\alpha_{i-1}, \alpha_{i}\right)=r$, and this is the case when $\alpha_{i}$ lies on the bound of $N\left(\alpha_{i-1}, r\right)$, for which the reasons of the case (a) are still true.

$$
\begin{aligned}
& \tilde{d}^{\prime}\left(\alpha_{i}, \tau_{i-1}\right)=\tilde{d}\left(\alpha_{i}, \tau_{i-1}\right)+r, \\
& \tilde{d}^{\prime}\left(\alpha_{i}, \tau_{i}\right)=\tilde{d}\left(\alpha_{i}, \tau_{i}\right)-r .
\end{aligned}
$$

This case is a combination of the situations, considered in cases (a) and (b). Q.E.D.

\section{REFERENCES}

[1] R. Dorfman, "The detection of defective members of large population”, Ann. Math. Stat., vol. 14, pp. 436-440, 1943.

[2] D. P. Bertsekas, Dynamic Programming and Optimal Control. Athena Scientific Publishers: Boston,1995.

[3] S. M. Ross, Introduction to Stochastic Dynamic Programming. Academic Press: New York, 1983.

[4] S. S. Brown, "Optimal search for a moving target in discrete time and space", Oper. Res., vol. 28, no. 6, pp. 1275-1289, 1980.

[5] L. D. Stone, Theory of Optimal Search. Academic Press: New York, 1975.

[6] J. N. Eagle, "The optimal search for a moving target when the search path is constrained", Oper. Res., vol. 32, pp. 1107-1115, 1984

[7] S. Singh, and V. Krishnamurthy, "The optimal search for Markovian target when the search path is constrained: the infinitehorizon case", IEEE Trans. Automat. Contr., vol. 48, no. 3, pp. 493-497, 2003.

[8] T. Ishida and R. E. Korf, "Moving target search", In: Proc. IJCAI Conference, 1991, pp. 204-210.

[9] T. Ishida and R. E. Korf, "Moving target search: a real-time search for changing goals", IEEE Trans. Pattern Anal., vol. 17, no. 6, pp. 609-619, 1995

[10] R. E. Korf, "Real-time heuristic search", Artif. Intell., vol. 42, no. 2-3, pp. 189-211, 1990.

[11] M. Shimbo and T. Ishida, "Controlling the learning process of realtime heuristic search", Artif. Intell., vol. 146, pp. 1-41, 2003.

[12] S. Koenig and G. Simmons, "Real-time search in non-deterministic domains", In: Proc. IJCAI Conference, 1995, pp. 1660-1667.

[13] S. Koenig and M. Likhachev, "Real-time adaptive A*", In Proc. AAMAS Conference, 2006, pp. 281-288.

[14] V. Bulitko and G. Lee, "Learning in real-time search: a unifying framework", J. Artif. Intell. Res., vol. 25, pp. 119-157, 2006. 
[15] V. Bulitko, N. Sturtevant, J. Lu and T. Yau, "Graph abstraction in real-time heuristic search", J. Artif. Intell. Res., vol. 30, 51-100, 2007.

[16] IRCL Research Group. Available, http://sites.google.com/a/ualber ta.ca/ircl/projects/rths [accessed on 14 January 2013].

[17] E. Kagan and I. Ben-Gal, "A group-testing algorithm with online informational learning", Trans. IIE, 2013.

[18] C. R. P. Hartmann, P. K. Varshney, K. G. Mehrotra and C. L. Gerberich, "Application of information theory to the construction of efficient decision trees", IEEE Trans. Inf. Theory, vol. 28, no. 4, pp. $565-577,1982$.

[19] J. Abrahams, "Parallelized Huffman and Hu-Tucker searching", IEEE Trans. Inf. Theory, vol. 40, no. 2, pp. 508-510, 1994.

[20] S. Zimmerman, "An optimal search procedure", Am. Math. Mon., vol. 66, pp. 690-693, 1959.

[21] V. A. Rokhlin. "Lectures on the entropy theory of measurepreserving transformations", Russ. Math. Surv+., vol. 22, pp. 1-52, 1967.

[22] T. M. Cover and J. A. Thomas, Elements of Information Theory. John Wiley \& Sons: New York, 1991.

[23] D. S. Ornstein, Ergodic Theory, Randomness, and Dynamical Systems. Yale University Press: New Haven, 1974.

[24] E. Kagan and I. Ben-Gal, "A MDP test for dynamic targets via informational measure”, In: Proc. IIE Research Meeting, 2007, pp. 39-53.

[25] E. Kagan and I. Ben-Gal, "An informational search for a moving target", In: Proc. IEEE 24-th Convention EEEI, 2006, pp. 153-155.

[26] Y. G. Sinai, Introduction to Ergodic Theory. Princeton University Press: Princeton, 1977.
[27] E. Kagan, G. Goren and I. Ben-Gal, "Probabilistic double-distance algorithm of search after static or moving target by autonomous mobile robot", In: Proc. IEEE 26-th Convention EEEI, 2010.

[28] E. Kagan, G. Goren and I. Ben-Gal, "Algorithm of search for static or moving target by autonomous mobile agent with erroneous sensor", In: Proc. IEEE 27-th Convention EEEI, 2012.

[29] I. Ben-Gal, E. Kagan and N. Shkolnik, "Constructing classification trees via data mining and design of experiments concepts", In: Proc. ENBIS Conference, 2008.

[30] Ben-Gal, N. Shkolnik and E. Kagan, "Greedy learning algorithms and their applications to decision trees", In: Proc. ENBIS Conference, 2007.

[31] G. Chernikhovsky, E. Kagan, G. Goren and I. Ben-Gal, "Path planning for sea vessel search using wideband sonar", In: Proc. IEEE 27-th Convention EEEI, 2012.

[32] D. J. White, Markov Decision Processes. John Wiley \& Sons: Chichester, 1993.

[33] J. M. Dobbie, "A two-cell model of search for a moving target", Oper. Res., vol. 22, no. 1, pp. 79-92, 1974.

[34] S. M. Pollock, "A simple model of search for a moving target", Oper. Res., vol. 18, pp. 883-903, 1970.

[35] E. Kagan, E. Salmona and I. Ben-Gal. "Probabilistic mobile robot with quantum decision-making", In: Proc. IEEE 25-th Convention EEEI, 2008, pp. 418-422.

[36] E. Kagan, I. Ben-Gal, N. Sharkov and O. Maimon, "Unsupervised zoning of scientific articles using Huffman trees", In: Proc. IEEE 25-th Convention EEEI, 2008, pp. 339-402.

[37] E. Kagan and I. Ben-Gal, "Search after a static target by multiple searchers by the use of informational distance measures", In: Proc. IE\&M Conference, 2010, p. 72. 